\title{
Modification of nucleon properties in nuclear matter and finite nuclei
}

\author{
W. Wen and H. Shen* \\ Department of Physics, Nankai University, Tianjin 300071, China
}

\begin{abstract}
We present a model for the description of nuclear matter and finite nuclei, and at the same time, for the study of medium modifications of nucleon properties. The nucleons are described as nontopological solitons which interact through the self-consistent exchange of scalar and vector mesons. The model explicitly incorporates quark degrees of freedom into nuclear many-body systems and provides satisfactory results on the nuclear properties. The present model predicts a significant increase of the nucleon radius at normal nuclear matter density. It is very interesting to see the nucleon properties change from the nuclear surface to the nuclear interior.
\end{abstract}

PACS numbers: 21.65.-f, 12.39.-x, 21.60.-n, 24.10.Jv

Keywords: Friedberg-Lee model, Nuclear matter, Finite nuclei

*Electronic address: songtc@nankai.edu.cn 


\section{INTRODUCTION}

One of the most interesting topics in nuclear physics is to study how the nucleon properties change in nuclear medium. So far, there are many experimental evidences indicating that the properties of the nucleon bound in nuclei are significantly modified from those of a free nucleon. The famous European Muon Collaboration (EMC) effect shows that the nucleon structure functions in nuclei deviate from those in a free nucleon [1]. Important evidences for medium modifications also come from recent polarization transfer experiments at the Thomas Jefferson National Accelerator Facility, which observed a difference in the electromagnetic form factors of a proton bound in a helium nucleus compared to a free one [2]. On the other hand, there are numerous theoretical works on the study of in-medium nucleon properties based on various models [3, 4, 5, 6]. At present, we are still far away from describing nucleons and nuclei in terms of quarks and gluons using quantum chromodynamics (QCD), which is believed to be the fundamental theory of strong interactions. Hence it is highly desirable to build models which could incorporate quark degrees of freedom and respect the established theories based on hadronic degrees of freedom.

The quark-meson coupling (QMC) model proposed by Guichon [7] can be considered as an extension of the extremely successful theoretical treatment of nuclear many-body systems, known as quantum hadrodynamics [8], to include the internal structure of the nucleon. The QMC model describes the nuclear system as nonoverlapping MIT bags in which the confined quarks interact through the self-consistent exchange of scalar and vector mesons in the mean-field approximation. In the QMC model, the quark structure of the nucleon plays a crucial role in the description of nuclear matter and finite nuclei [6]. It is also possible to study the medium modification of nucleon properties in the QMC model where the quark degrees of freedom are incorporated explicitly in the nuclear many-body system [9]. In the past decade, the QMC model has been extensively developed and applied with reasonable success to various nuclear phenomena $[\underline{6}, 10,11,12,13,14,15,16]$. There are also other models that incorporate quark degrees of freedom in the study of nuclear many-body systems. The quark mean-field (QMF) model [4] takes the constituent quark model for the nucleon instead of the MIT bag model, where the constituent quarks interact with the meson fields created by other nucleons. The QMF model has been successfully used for the description of nuclear matter, finite nuclei, and hypernuclei [17, 18, 19]. Recently, the QMF model has been extended to a model based on $S U(3)_{L} \times S U(3)_{R}$ symmetry and

scale invariance [20]. Using the Nambu-Jona-Lasinio model to describe the nucleon as a quark-diquark state, it is also possible to discuss the stability of nuclear matter based on the QMC idea [21]. The main advantage of these models is their simplicity and self-consistency in taking into account quark degrees of freedom in the study of nuclear many-body systems.

In this paper, we take the nontopological soliton bag model originally proposed by Friedberg and Lee [22], which is also called Friedberg-Lee model in the literature, for the description of nucleons in nuclear medium. In the Friedberg-Lee model, the nucleon is described 
as a bound state of three quarks in a nontopological soliton formed by a scalar field with nonlinear self-interactions. The Friedberg-Lee model has the benefits that it is manifestly covariant and it exhibits dynamical bag formation due to the coupling of quarks to the phenomenological scalar field. Furthermore, it includes the MIT bag as a special case. The soliton bag model has been extensively used to study the properties and structure of hadrons in free space [23, 24, 25, 26, 27, 28, 29, 30, 31]. It has also been applied to discuss the medium modification of nucleon properties [3, 32] and to study the dense matter properties within the Wigner-Seitz approximation [5]. In the present work, we develop a model to study the properties of nuclear matter and finite nuclei by describing the nuclear many-body system as a collection of nontopological soliton bags. The quarks inside the soliton bag couple not only to the scalar field that binds the quarks together into nucleons, but also to additional meson fields generated by the nuclear environment. The nucleons interact through the selfconsistent exchange of these mesons treated as classical fields in the spirit of the QMC and QMF models. Because in this model the soliton solution is significantly changed by the additional meson fields, it is possible to investigate the modification of nucleon properties in nuclear medium.

This paper is arranged as follows. In Sec. III, we briefly describe the Friedberg-Lee model for nucleons both in free space and in nuclear medium, and then discuss the properties of nuclear matter as a collection of nontopological soliton bags. In Sec. III, we show the properties of finite nuclei with the nucleons described as soliton bags, and also discuss how the nucleon properties change inside nuclei. Section IV is devoted to a summary.

\section{NUCLEAR MATTER AS NONTOPOLOGICAL SOLITON BAGS}

In this section, we first give a brief description of the Friedberg-Lee model for individual nucleons. Then we develop a model for nuclear matter regarded as a collection of nontopological soliton bags. This model enables us to investigate possible modifications of nucleon properties in nuclear medium.

\section{A. Free nucleon}

In the Friedberg-Lee model, a single nucleon is described as a bound state of three quarks in a nontopological soliton formed by a phenomenological scalar field with nonlinear selfinteractions. The Friedberg-Lee model in its simplest form is implemented through the effective Lagrangian density

$$
\mathcal{L}=\bar{\psi}\left(i \gamma_{\mu} \partial^{\mu}-m-g \phi\right) \psi+\frac{1}{2} \partial_{\mu} \phi \partial^{\mu} \phi-U(\phi)
$$

where $\psi$ denotes the quark field. The quark mass $m$ is usually taken to be zero for $u$ and $d$ quarks. $\phi$ is a color-singlet scalar field that may be interpreted as the phenomenological 
representation of quantum excitations of the self-interacting gluon field. The self-interaction of the scalar soliton field is described by the potential

$$
U(\phi)=\frac{a}{2 !} \phi^{2}+\frac{b}{3 !} \phi^{3}+\frac{c}{4 !} \phi^{4}+B .
$$

Here, the polynomial terminates in fourth order to ensure renormalizability. The constants $a, b$, and $c$ are fixed within a range so that $U(\phi)$ has a local minimum at $\phi=0$ and a global minimum at $\phi=\phi_{v}$. The constant $B$ is determined to make $U\left(\phi_{v}\right)=0$, and then the value $U(0)=B$ is to be identified with the bag constant or volume energy density of a cavity. We note that $\phi_{v}$ is the value of the soliton field in the physical vacuum where the quark gets a mass $\sim g \phi_{v}$ from its coupling with the soliton field. Inside the nucleon where valence quarks exist, the soliton field $\phi$ is reduced to be near zero that means the perturbative vacuum is restored. It is energetically favorable for three quarks to be localized in a cavity in the soliton field, referred to as the soliton bag, so that the nucleon appears as a bubble in the vacuum. In the mean-field approximation, the soliton field is treated as a classical field which is a time-independent $c$-number field $\phi(\mathbf{r})$. The quark field operator is expanded in a complete orthogonal set of Dirac spinor functions as $\psi=\sum_{k} b_{k} \psi_{k}$, where $b_{k}$ is the fermion annihilation operator. For a nucleon, the three valence quarks are in the lowest Dirac state $\psi_{0}$, then $\phi$ and $\psi_{0}$ satisfy the coupled differential equations

$$
\begin{aligned}
& (-i \vec{\alpha} \cdot \nabla+g \beta \phi) \psi_{0}=\epsilon_{0} \psi_{0}, \\
& -\nabla^{2} \phi+\frac{\partial U(\phi)}{\partial \phi}=-3 g \bar{\psi}_{0} \psi_{0} .
\end{aligned}
$$

The coupled equations have to be solved numerically. For the Dirac wave function of the $1 s_{1 / 2}$ quark, we use the notation

$$
\psi_{0}=\left(\begin{array}{c}
u(r) \\
i \vec{\sigma} \cdot \widehat{\mathbf{r}} v(r)
\end{array}\right) \chi
$$

with $\chi=\left(\begin{array}{l}1 \\ 0\end{array}\right)$ or $\left(\begin{array}{l}0 \\ 1\end{array}\right)$ being the Pauli spinor. The total energy of the nucleon is given by

$$
E=3 \epsilon_{0}+4 \pi \int d r r^{2}\left[\frac{1}{2}\left(\frac{d \phi}{d r}\right)^{2}+U(\phi)\right]
$$

The mean-square charge radius of the proton is given by

$$
\left\langle r^{2}\right\rangle=4 \pi \int d r r^{4}\left(u^{2}+v^{2}\right)
$$

and the proton magnetic moment is given by

$$
\mu_{p}=\frac{8 \pi}{3} \int d r r^{3} u v
$$

The ratio of the axial-vector to vector coupling constants is given by

$$
g_{A} / g_{V}=\frac{20 \pi}{3} \int d r r^{2}\left(u^{2}-\frac{1}{3} v^{2}\right) .
$$




\section{B. Center-of-mass correction}

There are several methods for calculating the center-of-mass (c.m.) correction to nucleon properties in the soliton bag model [24, 25, 26]. In this paper, we consider two approaches for the c.m. correction so that we can estimate how sensitive the results are to the c.m. correction approach used. First, we adopt an operationally simpler approach based on the relativistic energy-momentum relation to take into account the c.m. correction, which has been extensively discussed in Ref. [24]. The rest mass of the nucleon in this approach is given by

$$
M=\sqrt{E^{2}-\left\langle\mathbf{P}^{2}\right\rangle},
$$

where $\mathbf{P}$ is the total-momentum operator. The corrected root-mean-squared (rms) radius is given by

$$
r_{c}=\sqrt{\left[1-\frac{2 \epsilon_{0}}{E}+\frac{3 \epsilon_{0}^{2}}{E^{2}}\right]\left\langle r^{2}\right\rangle+\frac{3}{2 E^{2}}} .
$$

We also use the Peierls-Yoccoz projection technique to evaluate the c.m. correction to nucleon properties. Following the method described in Ref. [25], the rest mass of the nucleon is given by the expectation value of the Hamiltonian in the zero-momentum projected state. In this approach, the nucleon state is assumed to be the direct product of a soliton coherent state and a three-quark state

$$
|N\rangle=e^{\lambda A^{\dagger}} b_{1}^{\dagger} b_{2}^{\dagger} b_{3}^{\dagger}|0\rangle
$$

The soliton coherent state is given by

$$
e^{\lambda A^{\dagger}}|0\rangle=\prod_{\mathbf{k}} \exp \left[\sqrt{\frac{\omega_{k}}{2}} f_{\mathbf{k}} a_{\mathbf{k}}^{\dagger}\right]|0\rangle,
$$

where $a_{\mathbf{k}}^{\dagger}$ is the creation operator of the soliton field. The soliton field operator can be expanded in terms of $a_{\mathbf{k}}$ and $a_{\mathbf{k}}^{\dagger}$

$$
\phi(\mathbf{r})=\phi_{v}+(2 \pi)^{-3 / 2} \int d^{3} k \frac{1}{\sqrt{2 \omega_{k}}}\left(a_{\mathbf{k}} e^{i \mathbf{k} \cdot \mathbf{r}}+a_{\mathbf{k}}^{\dagger} e^{-i \mathbf{k} \cdot \mathbf{r}}\right) .
$$

The expectation value of the soliton field in the coherent state has exactly the properties of the mean-field value $\phi$ obtained by solving Eqs. (3) and (4). The soliton coherent state is localized, so the nucleon state has no definite momentum. To construct a momentum eigenstate, we use the Peierls-Yoccoz projection. The zero-momentum projected state is given by

$$
|\mathbf{P}=0\rangle=\int d^{3} X|\mathbf{X}\rangle
$$

where $|\mathbf{X}\rangle$ is a nucleon state localized at the point $\mathbf{X}$

$$
|\mathbf{X}\rangle=e^{\lambda A^{\dagger}(\mathbf{X})} b_{1}^{\dagger}(\mathbf{X}) b_{2}^{\dagger}(\mathbf{X}) b_{3}^{\dagger}(\mathbf{X})|0\rangle
$$


The operator $A^{\dagger}(\mathbf{X})$ can be written as

$$
\lambda A^{\dagger}(\mathbf{X})=\int d^{3} k \sqrt{\frac{\omega_{k}}{2}} f_{\mathbf{k}}(\mathbf{X}) a_{\mathbf{k}}^{\dagger}
$$

For a translationally invariant operator $O$, its expectation value in the zero-momentum projected state is given by

$$
\langle O\rangle=\frac{\langle\mathbf{P}=0|O| \mathbf{P}=0\rangle}{\langle\mathbf{P}=0 \mid \mathbf{P}=0\rangle}=\frac{\int d^{3} X d^{3} Y\langle\mathbf{X}|O| \mathbf{Y}\rangle}{\int d^{3} X d^{3} Y\langle\mathbf{X} \mid \mathbf{Y}\rangle}=\frac{\int d^{3} Z\left\langle-\frac{1}{2} \mathbf{Z}|O| \frac{1}{2} \mathbf{Z}\right\rangle}{\int d^{3} Z\left\langle-\frac{1}{2} \mathbf{Z} \mid \frac{1}{2} \mathbf{Z}\right\rangle},
$$

where $\mathbf{Z}=\mathbf{Y}-\mathbf{X}$. The normalization condition can be expressed as

$$
\left\langle-\frac{1}{2} \mathbf{Z} \mid \frac{1}{2} \mathbf{Z}\right\rangle=N_{\phi}(\mathbf{Z}) N_{q}(\mathbf{Z})^{3}
$$

where

$$
\begin{gathered}
N_{\phi}(\mathbf{Z})=\exp \left[\int d^{3} k \frac{\omega_{k}}{2} f_{\mathbf{k}}^{*}\left(-\frac{1}{2} \mathbf{Z}\right) f_{\mathbf{k}}\left(\frac{1}{2} \mathbf{Z}\right)\right], \\
N_{q}(\mathbf{Z})=\int d^{3} r \psi_{0}^{\dagger}\left(\mathbf{r}+\frac{1}{2} \mathbf{Z}\right) \psi_{0}\left(\mathbf{r}-\frac{1}{2} \mathbf{Z}\right) .
\end{gathered}
$$

Therefore, the nucleon mass is given by the expectation value of the Hamiltonian in the zero-momentum projected state

$$
M=\langle: H:\rangle=\left\langle: H_{q}+H_{q \phi}+H_{\phi}:\right\rangle
$$

These expectation values of normal-ordered products are

$$
\begin{gathered}
\left\langle: H_{q}+H_{q \phi}:\right\rangle=3 \int d^{3} Z N_{\phi}(\mathbf{Z}) N_{q}(\mathbf{Z})^{2} \frac{\int d^{3} r \psi_{0}^{\dagger}\left(\mathbf{r}+\frac{1}{2} \mathbf{Z}\right)[-i \vec{\alpha} \cdot \nabla+g \beta \bar{\phi}(\mathbf{r} ; \mathbf{Z})] \psi_{0}\left(\mathbf{r}-\frac{1}{2} \mathbf{Z}\right)}{\int d^{3} Z N_{\phi}(\mathbf{Z}) N_{q}(\mathbf{Z})^{3}}, \\
\left\langle: H_{\phi}:\right\rangle=\frac{\int d^{3} Z N_{\phi}(\mathbf{Z}) N_{q}(\mathbf{Z})^{3} \mathcal{E}_{\phi}(\mathbf{Z})}{\int d^{3} Z N_{\phi}(\mathbf{Z}) N_{q}(\mathbf{Z})^{3}},
\end{gathered}
$$

where

$$
\mathcal{E}_{\phi}(\mathbf{Z})=\int d^{3} r\left[\frac{1}{2} \bar{\pi}(\mathbf{r} ; \mathbf{Z})^{2}+\frac{1}{2}|\nabla \bar{\phi}(\mathbf{r} ; \mathbf{Z})|^{2}+U(\bar{\phi})\right] .
$$

The expectation values of the soliton field operators are given by

$$
\begin{gathered}
\bar{\phi}(\mathbf{r} ; \mathbf{Z})=\frac{1}{2}\left[\phi\left(\mathbf{r}-\frac{1}{2} \mathbf{Z}\right)+\phi\left(\mathbf{r}+\frac{1}{2} \mathbf{Z}\right)\right] \\
\bar{\pi}(\mathbf{r} ; \mathbf{Z})=-i(2 \pi)^{-3 / 2} \int d^{3} k \frac{\omega_{k}}{2}\left[f_{\mathbf{k}}\left(\frac{1}{2} \mathbf{Z}\right) e^{i \mathbf{k} \cdot \mathbf{r}}-f_{\mathbf{k}}^{*}\left(-\frac{1}{2} \mathbf{Z}\right) e^{-i \mathbf{k} \cdot \mathbf{r}}\right] .
\end{gathered}
$$

We note that the corrected rms radius in this approach has the same expression as given by Eq. (11) [26]. 


\section{Parameters in the Friedberg-Lee model}

In the Friedberg-Lee model, the parameters $a, b, c$, and $g$ are constrained by reproducing reasonable nucleon properties. We adjust the parameters to fit the nucleon mass $M=939$ $\mathrm{MeV}$ and rms radius $r_{c}=0.83 \mathrm{fm}$. In the present work, we take two sets of parameters using the c.m. correction given by Eq. (10). Set A: $a=0, b=-79.61 \mathrm{fm}^{-1}, c=780, g=13.7$ is characterized by $a=0$, where $U(\phi)$ has a inflection point at $\phi=0$. Set B: $a=69.945 \mathrm{fm}^{-2}$, $b=-1600 \mathrm{fm}^{-1}, c=12200, g=24.55$ is characterized by $B=0$, where the relationship among the parameters $b^{2}=3 a c$ is obtained by requiring $B=0$. The parameters in the Friedberg-Lee model have to be in a range where $U(\phi)$ has a local minimum at $\phi=0$ and a global minimum at $\phi=\phi_{v}$. Therefore, sets A and B correspond to the two limiting cases that can be seen in Fig. 11.

In order to compare the results with different c.m. correction approaches, we take another parameter set using the projection technique for the c.m. correction given by Eq. (22). Set $\mathrm{C}: a=19.08 \mathrm{fm}^{-2}, b=-335.02 \mathrm{fm}^{-1}, c=1961.0, g=24.55$ is also characterized by $B=0$. We use the same coupling constant $g$ in sets B and C. By comparing the results with these two parameter sets, we can estimate how sensitive the results are to the c.m. correction approach used.

We compute nucleon properties using these three parameter sets. The nucleon mass $M=939 \mathrm{MeV}$ and rms radius $r_{c}=0.83 \mathrm{fm}$ are obtained because they are constrains for the parameter sets. Set A gives the proton magnetic moment $\mu_{p}=2.80$ and the ratio of the axial-vector to vector coupling constants $g_{A} / g_{V}=0.87$. Set B predicts $\mu_{p}=2.77$ and $g_{A} / g_{V}=0.90$, whereas set $\mathrm{C}$ gives $\mu_{p}=2.85$ and $g_{A} / g_{V}=0.80$. We note that the experimental values are $\mu_{p}=2.79$ and $g_{A} / g_{V}=1.25$. It is shown that these three parameter sets in the Friedberg-Lee model can give reasonable results for nucleon properties in free space.

\section{Nuclear matter}

We develop a model for nuclear many-body system based on the Friedberg-Lee model in the spirit of the QMC model. Nuclear matter in this model is considered as a collection of nontopological soliton bags. The solitons interact through the self-consistent exchange of $\sigma, \omega$, and $\rho$ mesons that are treated as classical fields in the mean-field approximation. For a soliton embedded in nuclear matter, the quarks inside the nucleon couple not only to the soliton field $\phi$ which binds the quarks together into the nucleon, but also to additional meson fields $\sigma, \omega$, and $\rho$ generated by other nucleons in nuclear medium. We assume that the meson mean fields $\sigma, \omega$, and $\rho$ can be regarded as constants in uniform matter and the soliton field $\phi$ that serves to bind the quarks together does not participate in nucleon-nucleon interactions. Therefore, $\phi$ depends on spatial coordinates inside a nucleon, whereas $\sigma, \omega$, 
and $\rho$ are constants. With the presence of these additional meson fields in nuclear matter, the quark and soliton fields in the nucleon satisfy the coupled equations

$$
\begin{gathered}
\left(-i \vec{\alpha} \cdot \nabla+g \beta \phi+g_{\sigma}^{q} \beta \sigma+g_{\omega}^{q} \omega+g_{\rho}^{q} \tau_{3} \rho\right) \psi_{0}=\tilde{\epsilon}_{0} \psi_{0} \\
-\nabla^{2} \phi+\frac{\partial U(\phi)}{\partial \phi}=-3 g \bar{\psi}_{0} \psi_{0}
\end{gathered}
$$

where $g_{\sigma}^{q}, g_{\omega}^{q}$, and $g_{\rho}^{q}$ are the coupling constants of the $\sigma, \omega$, and $\rho$ mesons with quarks, respectively. We solve the coupled equations and calculate the in-medium nucleon properties analogously to the case of free nucleons. Here, the constant $\sigma$ field provides an additional scalar potential to the quarks and as a consequence changes the solutions $\psi_{0}$ and $\phi$ from those obtained by Eqs. (3) and (44). On the other hand, the $\omega$ and $\rho$ fields do not cause any changes of $\psi_{0}$ and $\phi$ except to shift the energy level by a constant vector potential, $\tilde{\epsilon}_{0}(\sigma, \omega, \rho)=\epsilon_{0}(\sigma)+g_{\omega}^{q} \omega+g_{\rho}^{q} \tau_{3} \rho$. Hence, $\psi_{0}$ and $\phi$ can be expressed as a function of the $\sigma$ mean field.

Analogously to the case of free nucleons, we use two approaches to take into account the c.m. correction for nucleons in nuclear matter. The first approach is based on the relativistic energy-momentum relation [33], in which the effective nucleon mass is given by

$$
M^{*}(\sigma)=\sqrt{E(\sigma)^{2}-\left\langle\mathbf{P}^{2}\right\rangle}
$$

where

$$
E(\sigma)=3 \epsilon_{0}(\sigma)+4 \pi \int d r r^{2}\left[\frac{1}{2}\left(\frac{d \phi}{d r}\right)^{2}+U(\phi)\right] .
$$

The second approach is based on the Peierls-Yoccoz projection technique as described in Sec. IIB. The effective nucleon mass is given by the expectation value of the Hamiltonian in the zero-momentum projected state

$$
M^{*}(\sigma)=\langle: H:\rangle=\frac{\langle\mathbf{P}=0|: H:| \mathbf{P}=0\rangle}{\langle\mathbf{P}=0 \mid \mathbf{P}=0\rangle},
$$

which can be calculated analogously to the case of free nucleons. We note that the quark wave function $\psi_{0}$ and the soliton field $\phi$ are altered by the $\sigma$ mean field in nuclear matter. Therefore, the calculated nucleon properties are different from those in free space. They can be expressed as functions of the $\sigma$ mean field. The density dependence of these quantities is obtained by a self-consistent determination of $\sigma$ at a given nuclear matter density. The prescription of Eq. (30) has also been used in the QMC and QMF models for removing the c.m. motion [9, 13, 17]. Another prescription used in the QMC model is to incorporate the c.m. correction into the parameter $z_{0}$ and assume that $z_{0}$ is independent of the matter density [11].

It is interesting to compare the two c.m. correction approaches used in this paper and investigate how the c.m. correction changes in nuclear matter. We define the c.m. energy 
of a nucleon in nuclear matter as $E_{\text {c.m. }}=E-M^{*}$, where $M^{*}$ is given by Eq. (30) in the first c.m. correction approach and by Eq. (32) in the second one. In Fig. 2, we show $E_{\text {c.m. }}$ as a function of the effective quark mass $m^{*}=g_{\sigma}^{q} \sigma$, which is proportional to the $\sigma$ mean field. The results of the first c.m. correction approach are shown by the solid lines, while those of the second approach are shown by the dashed lines. We have $m^{*}=0$ in free space and $m^{*} \sim-240 \mathrm{MeV}$ at normal nuclear matter density. It is seen that the variation of $E_{\text {c.m. }}$ in nuclear matter depends on the c.m. correction approach and the parameter set. As the density increases, $E_{\text {c.m. }}$. obtained in the first c.m. correction approach (solid lines) decreases in the upper panel and increases in the lower panel, whereas the results of the second approach (dashed lines) slowly decreases in both panels of Fig. 2. We find that the tendency of $E_{\text {c.m. }}$ with the second c.m. correction approach is quite similar to that shown in Fig. A.1 of Ref. [11] which is the results of a serious calculation for a relativistic harmonic oscillator potential. Therefore, the treatment for the c.m. correction by the projection technique is considered to be more reliable than the simple c.m. correction based on the relativistic energy-momentum relation.

To investigate in-medium nucleon properties and nuclear matter characteristics, we take a hybrid treatment for nuclear matter. The effective nucleon mass and couplings are obtained at the quark level, whereas the nucleon Fermi motion is treated at the hadron level. To perform a many-body calculation for nuclear matter, we start from the effective Lagrangian at the hadron level within the mean-field approximation

$$
\begin{aligned}
\mathcal{L}_{\mathrm{RMF}}= & \bar{\psi}\left[i \gamma_{\mu} \partial^{\mu}-M^{*}(\sigma)-g_{\omega} \gamma^{0} \omega-g_{\rho} \gamma^{0} \tau_{3} \rho\right] \psi \\
& -\frac{1}{2} m_{\sigma}^{2} \sigma^{2}+\frac{1}{2} m_{\omega}^{2} \omega^{2}+\frac{1}{2} m_{\rho}^{2} \rho^{2}
\end{aligned}
$$

where $\psi$ denotes the nucleon field. The effective nucleon mass $M^{*}(\sigma)$ is obtained in the Friedberg-Lee model, which has been given by Eq. (30) in the first c.m. correction approach and by Eq. (321) in the second one. The nonvanishing meson fields are replaced by their expectation values $\sigma=\langle\sigma\rangle, \omega=\left\langle\omega^{0}\right\rangle, \rho=\left\langle\rho^{03}\right\rangle$ that are constants in a static infinite nuclear matter. The nucleon-meson couplings are related to the quark-meson couplings as $g_{\omega}=3 g_{\omega}^{q}$ and $g_{\rho}=g_{\rho}^{q}$ [17]. From the Lagrangian given by Eq. (33), we obtain the equations of motion for nucleons and mesons in nuclear matter,

$$
\begin{gathered}
{\left[i \gamma_{\mu} \partial^{\mu}-M^{*}(\sigma)-g_{\omega} \gamma^{0} \omega-g_{\rho} \gamma^{0} \tau_{3} \rho\right] \psi=0,} \\
m_{\sigma}^{2} \sigma=-\frac{\partial M^{*}(\sigma)}{\partial \sigma}\langle\bar{\psi} \psi\rangle \\
m_{\omega}^{2} \omega=g_{\omega}\left\langle\bar{\psi} \gamma^{0} \psi\right\rangle \\
m_{\rho}^{2} \rho=g_{\rho}\left\langle\bar{\psi} \gamma^{0} \tau_{3} \psi\right\rangle .
\end{gathered}
$$

With $M^{*}(\sigma)$ obtained at the quark level, we solve the coupled equations self-consistently, and then calculate the nuclear matter properties and medium modifications of nucleon properties. In the present model, the quark-meson couplings $g_{\sigma}^{q}, g_{\omega}^{q}$, and $g_{\rho}^{q}$ are determined by 
reproducing the nuclear matter equilibrium density $\left(0.15 \mathrm{fm}^{-3}\right)$, energy per nucleon $(-16$ $\mathrm{MeV})$, and symmetry energy $(35 \mathrm{MeV})$. The meson masses are taken to be $m_{\sigma}=500 \mathrm{MeV}$, $m_{\omega}=783 \mathrm{MeV}$, and $m_{\rho}=770 \mathrm{MeV}$. We list in Table I the resulting nuclear matter properties corresponding to the parameter sets A, B, and C used in the Friedberg-Lee model. It is shown that the present model can provide a satisfactory description of nuclear matter properties.

Having determined the $\sigma$ mean field self-consistently in nuclear matter, we investigate in-medium nucleon properties with the quark wave function obtained by solving Eqs. (28) and (29). In Fig. 3 we plot the Dirac solutions $(u, v)$ and the soliton field $(\phi)$ as functions of the nucleon radius $(r)$ for the three parameter sets used. The results of the nucleon in free space are denoted by $u_{0}, v_{0}, \phi_{0}$, while those at the density $\rho_{0}$ and $2 \rho_{0}$ are denoted by $u_{1}, v_{1}$, $\phi_{1}$ and $u_{2}, v_{2}, \phi_{2}$, respectively. Here $\rho_{0}=0.15 \mathrm{fm}^{-3}$ is the normal nuclear matter density. By comparing the results in medium with those in free space, we find that the quark wave functions in nuclear matter are significantly different from those in free space. This leads to the medium modifications of nucleon properties because they are calculated using the quark wave functions. In the present model, the quark wave function depends on the $\sigma$ mean field, hence the density dependence of nucleon properties is obtained through the density dependence of the $\sigma$ mean field. It is interesting to compare the results obtained with the three parameter sets used. Set A gives stronger in-medium modifications than sets B and $\mathrm{C}$, especially at larger $r$. The results of set B is quite similar to those of set $\mathrm{C}$ because both of them are characterized by $B=0$. It is seen in Fig. 3 that the soliton field $(\phi)$ around the surface at high density in the case of set $\mathrm{A}$ is much broader than that in set $\mathrm{B}$. This yields more leakage of the quark wave function in set $\mathrm{A}$ than in set B. It may lead to the difference in the density dependence of nucleon properties, such as $r_{c}$ and $g_{A} / g_{V}$, because they are sensitive to the behavior of the quark wave function at large $r$. By comparing with the sharp boundary of the MIT bag model, we find that set B is closer to the MIT bag model than set A. Therefore, the results of set B are rather close to those of the MIT bag model. This indicates that the surface behavior of the confinement potential plays an important role in determining nucleon properties.

In Fig. 4, we present the ratio of the effective nucleon mass in nuclear matter to that in free space, $M^{*} / M$, as a function of nuclear matter density $\rho$. The solid and dashed lines correspond to the cases of set $\mathrm{A}$ and set $\mathrm{B}$ where the effective nucleon mass $M^{*}$ is given by Eq. (30) using the first c.m. correction approach. The dotted line corresponds to the case of set $\mathrm{C}$ where $M^{*}$ is given by Eq. (32) using the second c.m. correction approach. We note that the effective nucleon mass in the present model is calculated at the quark level, which is not a simple linear function of $\sigma$ as given in the Walecka model [8]. It is more like the characteristics of the QMC model. As shown in Fig. 4, the effective nucleon mass decreases with increasing density, and the results depend on the parameter set used. The large difference between sets $\mathrm{B}$ and $\mathrm{C}$ is mainly due to the different c.m. correction approaches used in these two cases. The first c.m. correction approach used in the case of 
set B provides a strong increase of $E_{\text {c.m. }}$ in nuclear matter (see solid line in the lower panel of Fig. 2), whereas the second c.m. correction approach used in the case of set $\mathrm{C}$ gives a weak decrease of $E_{\text {c.m. }}$ (see dashed line in Fig. 2). This leads to the difference in $M^{*}$ between sets $\mathrm{B}$ and $\mathrm{C}$ due to the relation $M^{*}=E-E_{\text {c.m. }}$. Therefore, the drop of $M^{*}$ in set $\mathrm{B}$ is much more rapid than that in set $\mathrm{C}$. On the other hand, the difference between sets $\mathrm{A}$ and $\mathrm{B}$ can be understood as a consequence of the different behaviors of $E_{\text {c.m. }}$ in these two cases (compare solid lines in the upper and lower panels of Fig. 2), though the first c.m. correction approach is used in both sets $\mathrm{A}$ and $\mathrm{B}$.

We show in Fig. 5 the ratio of the nucleon rms radius in nuclear matter to that in free space, $r_{c}^{*} / r_{c}$, as a function of nuclear matter density $\rho$. It is very interesting to see the expansion of the nucleon size in medium. We find the nucleon rms radius increases by about $10-16 \%$ at normal nuclear matter density. This result is quite different from those obtained in other models. For example, the QMC model predicts only 1-3\% enhancement in the nucleon rms radius at normal nuclear matter density [10], and the QMF model gives about $5-9 \%$ increase [17]. The chiral quark-soliton model predicts a $2.4 \%$ enhancement, while the swelling constrained by quasielastic inclusive electron-nucleus scattering data is less than $6 \%$ [34]. We note that although the Friedberg-Lee model can give similar quark distributions and nucleon properties to those obtained in the MIT bag model in free space, the quarks satisfy different equations and boundary conditions in these two models, and therefore the swelling of the nucleon rms radius could be quite different between the QMC model and the present calculation. In Fig. 6, we present the ratio of the proton magnetic moment in nuclear matter to that in free space, $\mu_{p}^{*} / \mu_{p}$, as a function of nuclear matter density $\rho$. It is shown that the results depend on the parameter set used. The difference between different parameter sets increases with increasing density. Set A gives nearly the same low-density behavior as set $\mathrm{B}$, but rather different results at high density. The enhancement in set $\mathrm{C}$ is smaller than those in sets A and B. We show in Fig. 7 the ratio of the axial-vector to vector coupling constants in nuclear matter $\left(g_{A} / g_{V}\right)^{*}$ to that in free space $g_{A} / g_{V}$ as a function of nuclear matter density $\rho$. The results of set A decrease slightly at lower densities, and then increase at higher densities. On the other hand, the results of set B drop significantly with increasing density. The results of set $\mathrm{C}$ is between sets $\mathrm{A}$ and $\mathrm{B}$. At normal nuclear matter density, we obtain $\left(g_{A} / g_{V}\right)^{*} /\left(g_{A} / g_{V}\right) \simeq 0.98$ for set $\mathrm{A},\left(g_{A} / g_{V}\right)^{*} /\left(g_{A} / g_{V}\right) \simeq 0.87$ for set $\mathrm{B}$, and $\left(g_{A} / g_{V}\right)^{*} /\left(g_{A} / g_{V}\right) \simeq 0.94$ for set C. It is obvious that medium modifications of nucleon properties in this work depend on the parameter set used in the Friedberg-Lee model. Because set A gives stronger in-medium modifications of the quark wave functions than sets B and $\mathrm{C}$ as shown in Fig. 3, we obtain larger increases in $r_{c}^{*}$ and $\mu_{p}^{*}$ with set A, as shown in Figs. 5 and 6. On the other hand, the difference in $\left(g_{A} / g_{V}\right)^{*}$ shown in Fig. 7 could be due to the competition between changes of $u$ and $v$ according to Eq. (9). 


\section{PROPERTIES OF FINITE NUCLEI}

In this section, we extend the present model to study the properties of finite nuclei and the modification of nucleon properties in a nucleus. The nucleus is described as a collection of nontopological soliton bags that interact through the self-consistent exchange of $\sigma, \omega$, and $\rho$ mesons. In principle, these meson mean fields are functions of the spatial coordinates in the nucleus, but it is rather complicated if the variation of these quantities over the small nucleon volume is taken into account. Therefore, we take some suitably averaged form for the meson mean fields in order to make the numerical solution feasible. We use the local density approximation which replace the meson mean fields by their value at the center of the nucleon and neglect the spatial variation of the mean fields over the small nucleon volume [10, 14, 17]. The equations of motion for nucleons and mesons in a spherically symmetric nucleus are given by

$$
\begin{gathered}
{\left[i \gamma_{\mu} \partial^{\mu}-M^{*}(\sigma)-g_{\omega} \gamma^{0} \omega-g_{\rho} \gamma^{0} \tau_{3} \rho-e \frac{\left(1+\tau_{3}\right)}{2} \gamma^{0} A\right] \psi=0} \\
\left(-\Delta+m_{\sigma}^{2}\right) \sigma=-\frac{\partial M^{*}(\sigma)}{\partial \sigma}\langle\bar{\psi} \psi\rangle, \\
\left(-\Delta+m_{\omega}^{2}\right) \omega=g_{\omega}\left\langle\bar{\psi} \gamma^{0} \psi\right\rangle, \\
\left(-\Delta+m_{\rho}^{2}\right) \rho=g_{\rho}\left\langle\bar{\psi} \gamma^{0} \tau_{3} \psi\right\rangle, \\
-\Delta A=e\left\langle\bar{\psi} \frac{\left(1+\tau_{3}\right)}{2} \gamma^{0} \psi\right\rangle,
\end{gathered}
$$

where the mean fields are functions of the radial coordinate of the nucleon center in the nucleus. We solve the preceding equations self-consistently with the effective nucleon mass obtained at the quark level.

We present the numerical results of several spherical nuclei. In Table II, the calculated binding energies per nucleon and rms charge radii are compared with the experimental values [35]. By comparing the results of sets $\mathrm{A}, \mathrm{B}$, and $\mathrm{C}$, we find that the binding energies of set $\mathrm{C}$ are larger than those of sets $\mathrm{A}$ and $\mathrm{B}$. This is mainly because set $\mathrm{C}$ gives smaller incompressibility $(K=184 \mathrm{MeV})$ than sets $\mathrm{A}(K=302 \mathrm{MeV})$ and $\mathrm{B}(K=384 \mathrm{MeV})$ when they have the same saturation density $\left(\rho_{0}=0.15 \mathrm{fm}^{-3}\right)$ and energy per particle $(E / A=-16 \mathrm{MeV})$ as shown in Table I. We list the calculated single-particle energies and spin-orbit splittings in Tables III and IV, and compare with the experimental data taken from Ref. [36]. It is seen that all calculated spin-orbit splittings are smaller than the experimental data, while set $\mathrm{C}$ gives the smallest values among the three parameter sets. This is due to the large effective nucleon masses obtained in the present model as shown in Table I. According to analysis with many quantum-hadrodynamics models, there exists a tight correlation between the spin-orbit splitting of finite nuclei and the effective nucleon mass in nuclear matter at saturation density, and the spin-orbit splitting increases 
with decreasing $M^{*}$. It is known that $M^{*} / M \sim 0.6$ is required in order to reproduce the empirical spin-orbit splittings of finite nuclei. This is the reason why we get small spin-orbit splittings in the present model and the smallest values in set $\mathrm{C}$. This shortage might be improved by including nonlinear meson self-interactions as taken in the QMC model [14]. We plot in Fig. 8 the resulting charge density distributions for ${ }^{208} \mathrm{~Pb}$ and compare with the experimental values [37]. As seen in this figure, the calculated results are in good agreement with the experimental values, and there is no explicit difference between the three parameter sets.

It is also possible to investigate the modification of nucleon properties in finite nuclei. Using the local density approximation, the nucleon properties at the radial coordinate $r$ in a nucleus are obtained through the values of $\sigma(r)$, because the nucleon properties in this model are functions of the $\sigma$ mean field obtained at the quark level. In Figs. 9 and 10, we show the ratios of the proton rms radius and magnetic moment in ${ }^{208} \mathrm{~Pb}$ to those in free space as functions of the radius $r$. It is found that the proton radius and magnetic moment increase significantly at the center of ${ }^{208} \mathrm{~Pb}$. These quantities decrease to the values in free space from the center to the surface of the nucleus. It is shown that the results depend on the parameter set used, which is consistent with the results of nuclear matter shown in Figs. 5 and 6 .

\section{CONCLUSION}

By treating the nucleons as nontopological soliton bags, we have proposed a model for the description of nuclear matter and finite nuclei, and at the same time for the study of medium modifications of nucleon properties. The nontopological soliton bag model, proposed by Friedberg and Lee, exhibits a dynamical bag formation due to the coupling of quarks to the phenomenological scalar field $\phi$. The quarks inside the soliton bag couple not only to the scalar field $\phi$ that binds the quarks together into nucleons, but also to additional meson fields $\sigma, \omega$, and $\rho$ generated by the nuclear environment. The nucleons interact through the self-consistent exchange of $\sigma, \omega$, and $\rho$ meson fields that are treated as classical fields in the mean-field approximation. This model enables us to investigate the medium modification of nucleon properties because the soliton bag is significantly influenced by the additional meson fields in nuclear medium.

We have considered two approaches for the c.m. correction to nucleon properties so that we can estimate how sensitive the results are to the c.m. correction approach used. We have adopted three parameter sets in the Friedberg-Lee model that are constrained by reproducing free nucleon properties. The quark-meson coupling constants are fitted to reproduce the empirical saturation properties of nuclear matter. The present model can provide a reasonable description of nuclear matter. We have found that the properties of the nucleon are significantly modified in nuclear medium. At normal nuclear matter density, 
the nucleon radius increases by about 10-16\%, while the proton magnetic moment increases by about $11-16 \%$. We have applied the present model to study the properties of spherical nuclei and found that it could give a reasonable description of the ground state properties of finite nuclei. It is very interesting to see the nucleon properties change from the nuclear surface to the nuclear interior. The present model incorporates explicit quark degrees of freedom into nuclear many-body systems. It is notable that the quark structure of the nucleon plays a crucial role in the description of nuclear matter and finite nuclei.

\section{Acknowledgments}

This work was supported in part by the National Natural Science Foundation of China (No. 10675064).

[1] J. J. Aubert et al., Phys. Lett. B123, 275 (1983).

[2] S. Strauch et al., Phys. Rev. Lett. 91, 052301 (2003).

[3] L. S. Celenza, A. Rosenthal, and C. M. Shakin, Phys. Rev. C 31, 232 (1985).

[4] H. Toki, U. Meyer, A. Faessler, and R. Brockmann, Phys. Rev. C 58, 3749 (1998).

[5] N. Barnea and T. S. Walhout, Nucl. Phys. A677, 367 (2000).

[6] K. Saito, K. Tsushima, and A. W. Thomas, Prog. Part. Nucl. Phys. 58, 1 (2007).

[7] P. A. M. Guichon, Phys. Lett. B200, 235 (1988).

[8] B. D. Serot and J. D. Walecka, Adv. Nucl. Phys. 16, 1 (1986).

[9] K. Saito and A. W. Thomas, Phys. Rev. C 51, 2757 (1995).

[10] K. Saito, K. Tsushima, and A. W. Thomas, Nucl. Phys. A609, 339 (1996).

[11] P. A. M. Guichon, K. Saito, E. Rodionov, and A. W. Thomas, Nucl. Phys. A601, 349 (1996).

[12] P. A. M. Guichon, K. Saito, and A. W. Thomas, Aust. J. Phys. 50, 115 (1997).

[13] H. Muller and B. K. Jennings, Nucl. Phys. A626, 966 (1997).

[14] H. Muller and B. K. Jennings, Nucl. Phys. A640, 55 (1998).

[15] S. Pal, M. Hanauske, I. Zakout, H. Stocker, and W. Greiner, Phys. Rev. C 60, 015802 (1999).

[16] P. K. Panda, D. P. Menezes, and C. Providencia, Phys. Rev. C 69, 025207 (2004).

[17] H. Shen and H. Toki, Phys. Rev. C 61, 045205 (2000).

[18] H. Shen and H. Toki, Nucl. Phys. A707, 469 (2002).

[19] H. Shen and H. Toki, Phys. Rev. C 71, 065208 (2005).

[20] P. Wang, H. Guo, Z. Y. Zhang, Y. W. Yu, R. K. Su, and H. Q. Song, Nucl. Phys. A705, 455 (2002); P. Wang, S. Lawley, D. B. Leinweber, A. W. Thomas, and A. G. Williams, Phys. Rev. C 72, 045801 (2005).

[21] W. Bentz and A. W. Thomas, Nucl. Phys. A696, 138 (2001).

[22] R. Friedberg and T. D. Lee, Phys. Rev. D 15, 1694 (1977); 16, 1096 (1977); 18, 2623 (1978). 
[23] R. Goldflam and L. Wilets, Phys. Rev. D 25, 1951 (1982).

[24] J. L. Dethier, R. Goldflam, E. M. Henley, and L. Wilets, Phys. Rev. D 27, 2191 (1983).

[25] E. G. Lubeck, M. C. Birse, E. M. Henley, and L. Wilets, Phys. Rev. D 33, 234 (1986).

[26] E. G. Lubeck, E. M. Henley, and L. Wilets, Phys. Rev. D 35, 2809 (1987).

[27] Q. Haider and L. C. Liu, J. Phys. G 12, L75 (1986).

[28] Enke Wang, Jiarong Li, and Lianshou Liu, Phys. Rev. D 41, 2288 (1990).

[29] M. C. Birse, Prog. Part. Nucl. Phys. 25, 1 (1990).

[30] T. D. Lee and Y. Pang, Phys. Rep. 221, 251 (1992).

[31] C. Wu, W. L. Qian, and R. K. Su, Phys. Rev. C 72, 035205 (2005); H. Mao, R. K. Su, and W. Q. Zhao, ibid. 74, 055204 (2006).

[32] E. Naar and M. C. Birse, J. Phys. G 19, 555 (1993).

[33] S. Fleck, W. Bentz, K. Shimizu, and K. Yazaki, Nucl. Phys. A510, 731 (1990).

[34] J. R. Smith and G. A. Miller, Phys. Rev. C 70, 065205 (2004).

[35] Y. Sugahara and H. Toki, Nucl. Phys. A579, 557 (1994).

[36] X. Campi and D. W. Sprung, Nucl. Phys. A194, 401 (1972).

[37] H. de Vries, C. W. de Jager, and C. de Vries, At. Data Nucl. Data Tables 36, 495 (1987). 
TABLE I: The nuclear matter properties in the present model with the parameter sets A, B, and C. The saturation density and the energy per particle are denoted by $\rho_{0}$ and $E / A$, the symmetry energy by $a_{\text {sym }}$, the incompressibility by $K$, and the effective mass by $M^{*}$.

\begin{tabular}{cccccc}
\hline \hline & $\begin{array}{c}\rho_{0} \\
\left(\mathrm{fm}^{-3}\right)\end{array}$ & $\begin{array}{c}E / A \\
(\mathrm{MeV})\end{array}$ & $\begin{array}{c}a_{\text {sym }} \\
(\mathrm{MeV})\end{array}$ & $\begin{array}{c}K \\
(\mathrm{MeV})\end{array}$ & $M^{*} / M$ \\
\hline Set A & 0.15 & -16.0 & 35 & 302 & 0.81 \\
Set B & 0.15 & -16.0 & 35 & 384 & 0.72 \\
Set C & 0.15 & -16.0 & 35 & 184 & 0.87 \\
\hline \hline
\end{tabular}

TABLE II: The binding energy per nucleon $E / A$ and the rms charge radius $R_{c}$ for ${ }^{40} \mathrm{Ca},{ }^{90} \mathrm{Zr}$, and ${ }^{208} \mathrm{~Pb}$.

\begin{tabular}{|c|c|c|c|c|c|c|c|c|}
\hline & \multicolumn{4}{|c|}{$E / A(\mathrm{MeV})$} & \multicolumn{4}{|c|}{$R_{c}(\mathrm{fm})$} \\
\hline & Set A & Set B & Set $C$ & Expt. & Set A & Set B & Set $\mathrm{C}$ & Expt. \\
\hline${ }^{40} \mathrm{Ca}$ & 8.53 & 7.66 & 9.35 & 8.55 & 3.42 & 3.46 & 3.38 & 3.45 \\
\hline${ }^{90} \mathrm{Zr}$ & 8.36 & 7.81 & 8.97 & 8.71 & 4.26 & 4.27 & 4.24 & 4.26 \\
\hline${ }^{208} \mathrm{~Pb}$ & 7.48 & 7.13 & 7.95 & 7.87 & 5.52 & 5.50 & 5.54 & 5.50 \\
\hline
\end{tabular}

TABLE III: The single-particle energies of proton $(p)$ and neutron $(n)$ for ${ }^{40} \mathrm{Ca}$. The experimental data are taken from Ref. [36]. All energies are in MeV.

\begin{tabular}{|c|c|c|c|c|c|c|c|c|}
\hline \multirow[b]{2}{*}{ Shell } & \multicolumn{2}{|c|}{ Set A } & \multicolumn{2}{|c|}{ Set B } & \multicolumn{2}{|c|}{ Set $\mathrm{C}$} & \multicolumn{2}{|c|}{ Expt. } \\
\hline & $p$ & $n$ & $p$ & $n$ & $p$ & $n$ & $p$ & $n$ \\
\hline $1 s_{1 / 2}$ & 35.6 & 43.7 & 37.8 & 45.9 & 34.7 & 42.8 & $50 \pm 11$ & 50.0 \\
\hline $1 p_{3 / 2}$ & 24.6 & 32.4 & 25.7 & 33.5 & 24.4 & 32.2 & $34 \pm 6$ & 30.0 \\
\hline $1 p_{1 / 2}$ & 23.5 & 31.3 & 23.6 & 31.4 & 23.7 & 31.6 & $34 \pm 6$ & 27.0 \\
\hline $1 d_{5 / 2}$ & 12.5 & 20.0 & 12.8 & 20.3 & 12.6 & 20.2 & 15.5 & 21.9 \\
\hline $1 d_{3 / 2}$ & 10.4 & 18.0 & 9.3 & 16.8 & 11.4 & 19.0 & 8.3 & 15.6 \\
\hline $2 s_{1 / 2}$ & 8.1 & 15.6 & 7.9 & 15.2 & 8.4 & 15.9 & 10.9 & 18.2 \\
\hline
\end{tabular}


TABLE IV: The spin-orbit splittings of proton $\left(\Delta E_{p}\right)$ and neutron $\left(\Delta E_{n}\right)$ for ${ }^{40} \mathrm{Ca}$ and ${ }^{208} \mathrm{~Pb}$. The experimental data are taken from Ref. [36]. All quantities are in $\mathrm{MeV}$.

\begin{tabular}{lcccc}
\hline \hline & \multicolumn{2}{c}{${ }^{40} \mathrm{Ca}$} & \multicolumn{2}{c}{${ }^{208} \mathrm{~Pb}$} \\
\cline { 2 - 5 } \cline { 4 - 5 } & $\Delta E_{p}$ & $\Delta E_{n}$ & $\Delta E_{p}$ & $\Delta E_{n}$ \\
& $\left(1 d_{5 / 2}-1 d_{3 / 2}\right)$ & $\left(1 d_{5 / 2}-1 d_{3 / 2}\right)$ & $\left(1 g_{9 / 2}-1 g_{7 / 2}\right)$ & $\left(2 f_{7 / 2}-2 f_{5 / 2}\right)$ \\
\hline Set A & 2.0 & 2.0 & 1.2 & 0.7 \\
Set B & 3.5 & 3.5 & 2.2 & 1.3 \\
Set C & 1.1 & 1.1 & 0.6 & 0.4 \\
Expt. & 7.2 & 6.3 & 4.0 & 1.8 \\
\hline \hline
\end{tabular}

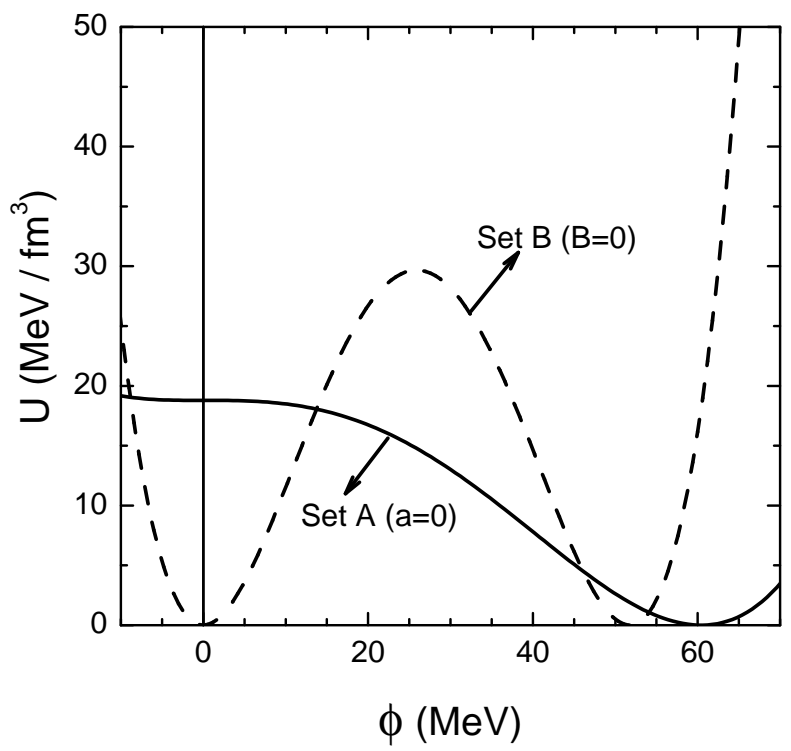

FIG. 1: The self-interaction of the soliton field as a function of $\phi$ for the two parameter sets characterized by $a=0$ and $B=0$. 


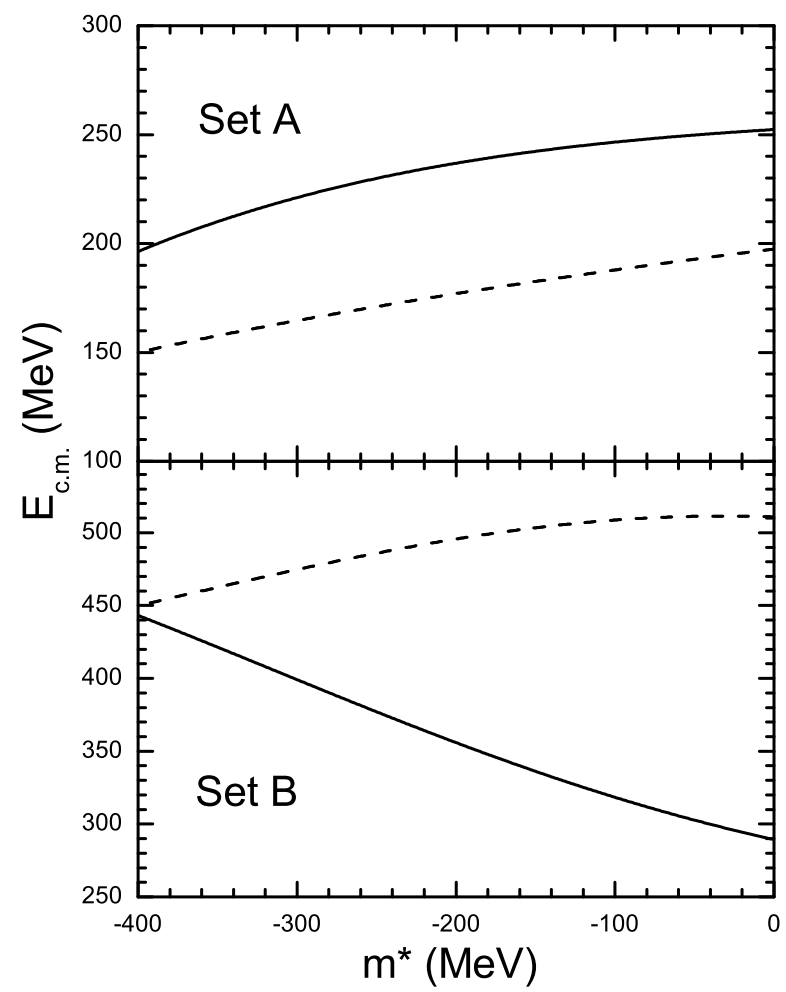

FIG. 2: The c.m. energy of the nucleon in nuclear matter, $E_{\text {c.m. }}=E-M^{*}$, as a function of the effective quark mass, $m^{*}=g_{\sigma}^{q} \sigma$. The solid lines show the results of the first c.m. correction approach where $M^{*}$ is given by Eq. (30). The dashed lines show the results of the second c.m. correction approach with $M^{*}$ given by Eq. (32). We note that $m^{*}=0$ in free space and $m^{*} \sim-240$ $\mathrm{MeV}$ at normal nuclear matter density. 


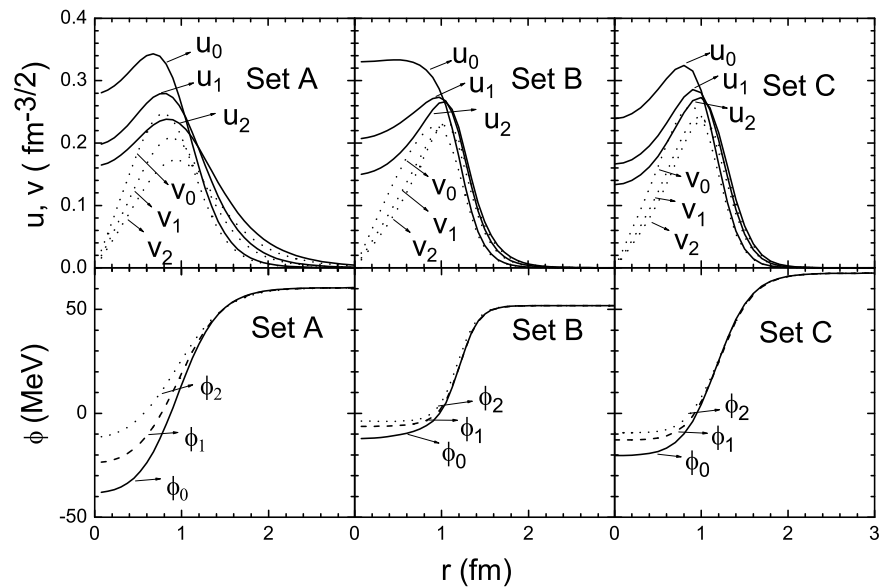

FIG. 3: The quark wave functions $(u, v)$ and the scalar field $\phi$ as a function of the nucleon radius $(r)$ with the three parameter sets. The results of the nucleon in free space are denoted by $u_{0}, v_{0}$ and $\phi_{0}$. The results at the density $\rho_{0}$ and $2 \rho_{0}$ are denoted by $u_{1}, v_{1}, \phi_{1}$ and $u_{2}, v_{2}, \phi_{2}$, respectively, where $\rho_{0}=0.15 \mathrm{fm}^{-3}$ is the normal nuclear matter density.

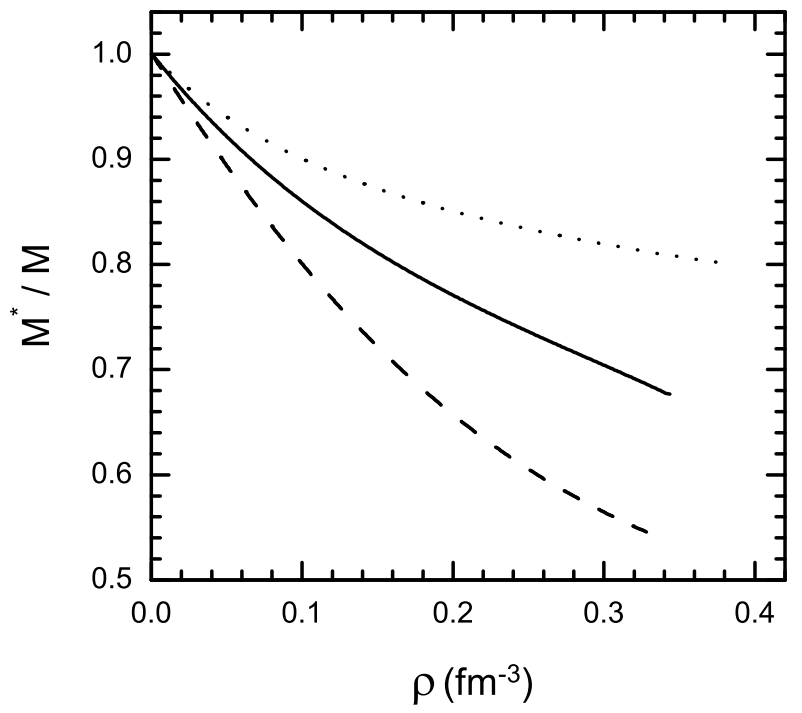

FIG. 4: The ratio of the effective nucleon mass in nuclear matter to that in free space, $M^{*} / M$, as a function of nuclear matter density $\rho$. The results with the parameter sets $\mathrm{A}, \mathrm{B}$, and $\mathrm{C}$ are shown by the solid, dashed, and dotted lines, respectively. 


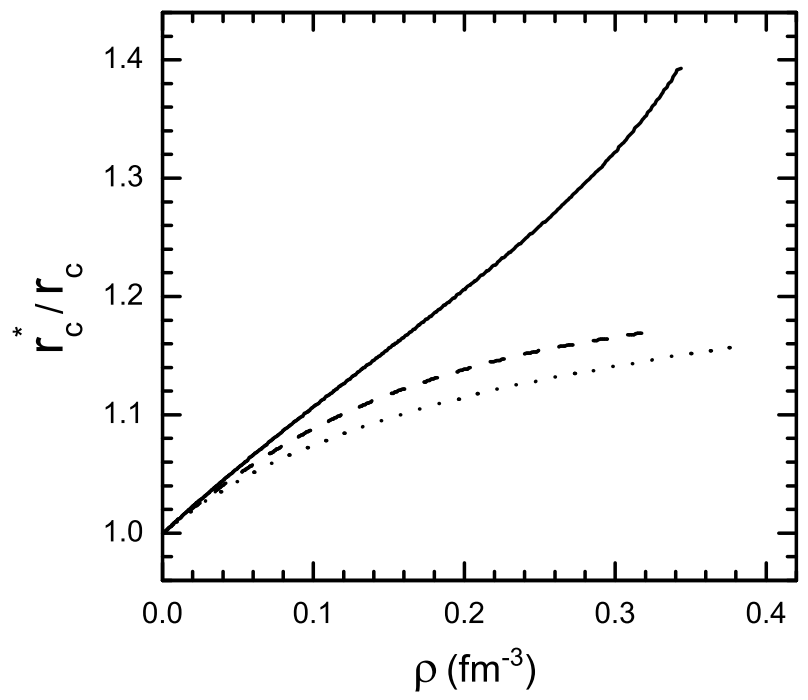

FIG. 5: The ratio of the nucleon rms radius in nuclear matter to that in free space, $r_{c}^{*} / r_{c}$, as a function of nuclear matter density $\rho$. The lines are labeled as in Fig. 4.

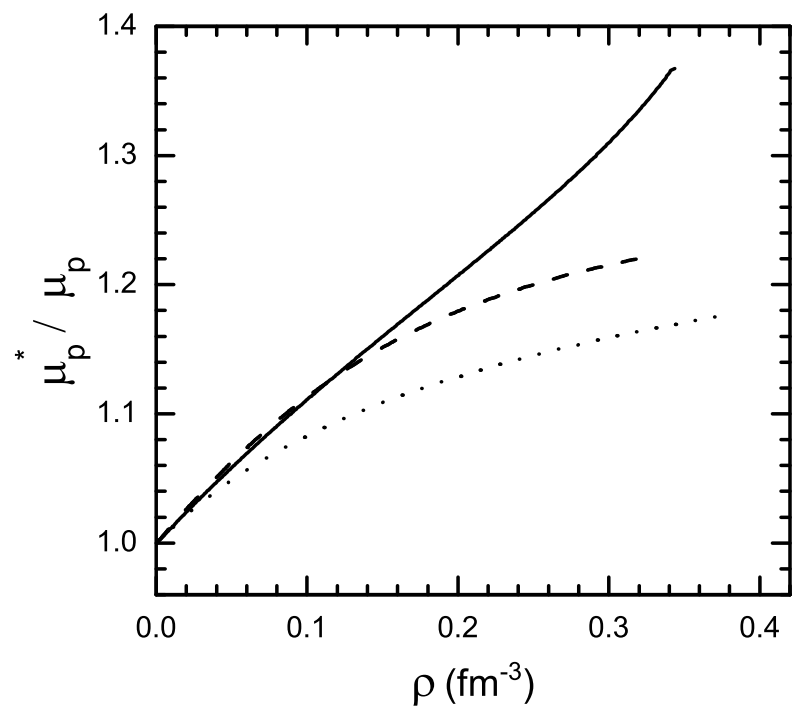

FIG. 6: The ratio of the proton magnetic moment in nuclear matter to that in free space, $\mu_{p}^{*} / \mu_{p}$, as a function of nuclear matter density $\rho$. The lines are labeled as in Fig. 4 . 


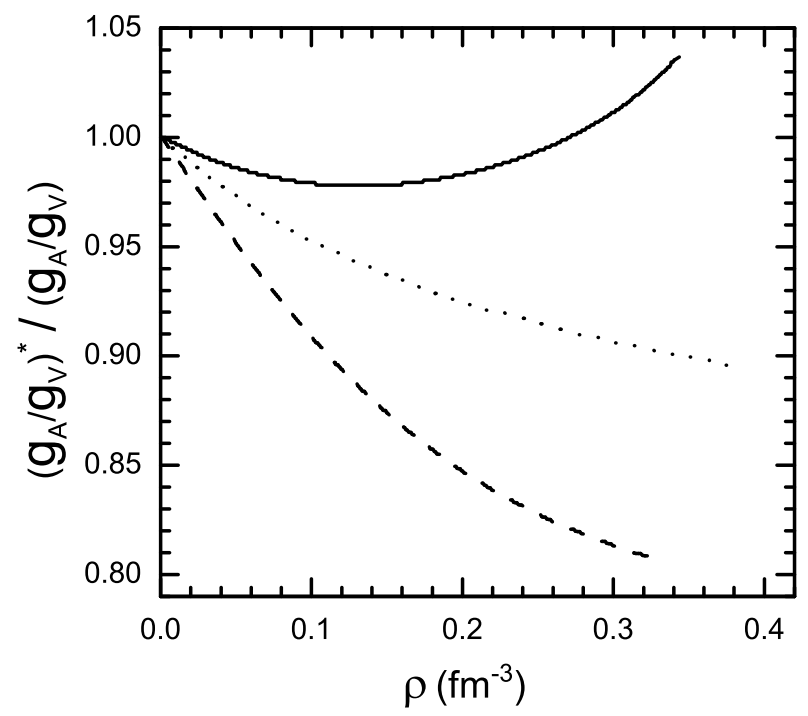

FIG. 7: The ratio of the axial-vector to vector coupling constants in nuclear matter $\left(g_{A} / g_{V}\right)^{*}$ to that in free space $g_{A} / g_{V}$ as a function of nuclear matter density $\rho$. The lines are labeled as in

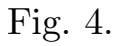

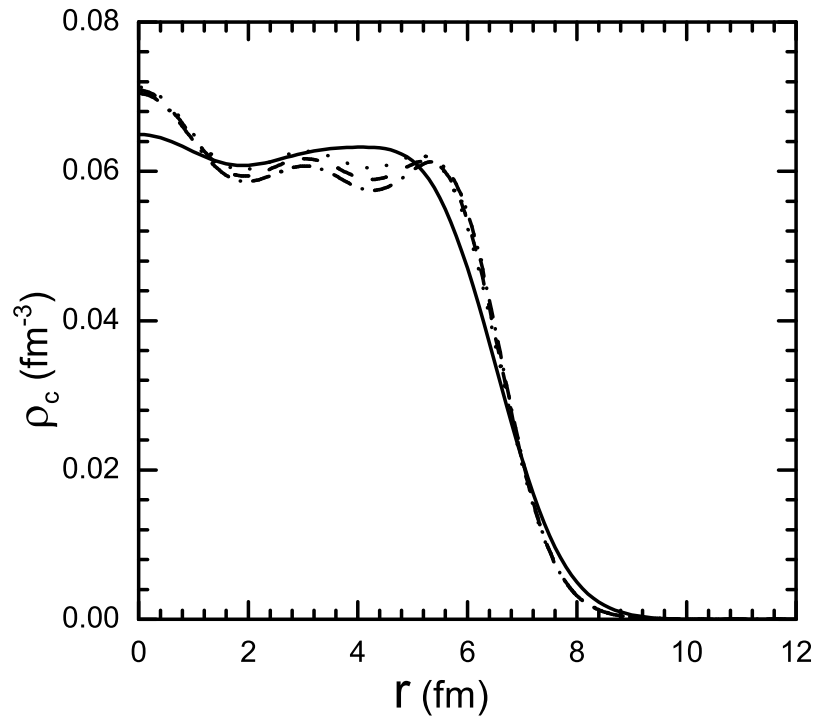

FIG. 8: The charge density distributions for ${ }^{208} \mathrm{~Pb}$ compared with the experimental data (solid line) [37]. The results with the parameter sets A, B, and C are shown by the dashed, dotted, and dot-dashed lines, respectively. 


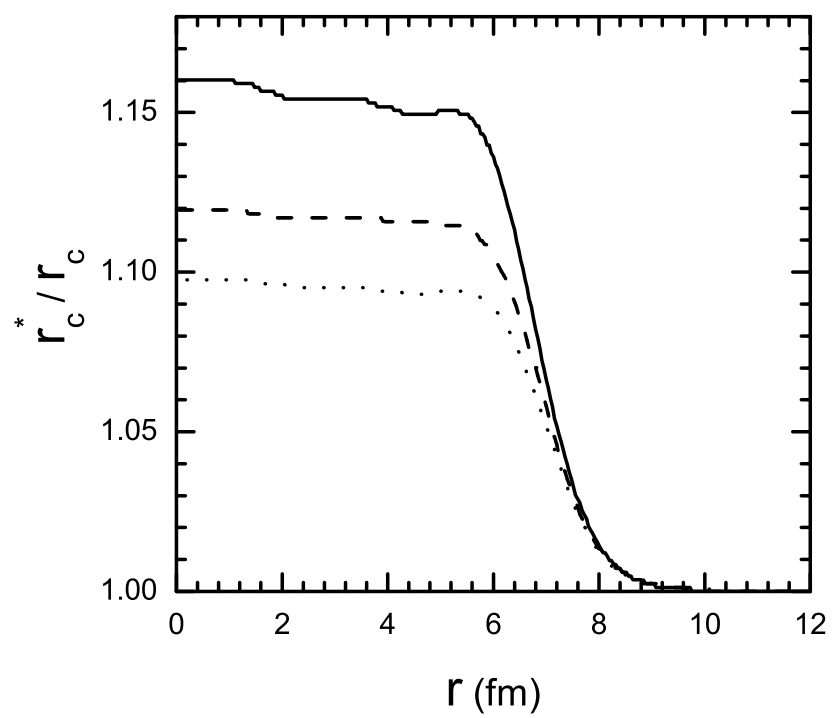

FIG. 9: The ratio of the proton rms radius in ${ }^{208} \mathrm{~Pb}$ to that in free space as a function of radial coordinate $r$. The results with the parameter sets A, B, and C are shown by the solid, dashed, and dotted lines, respectively.

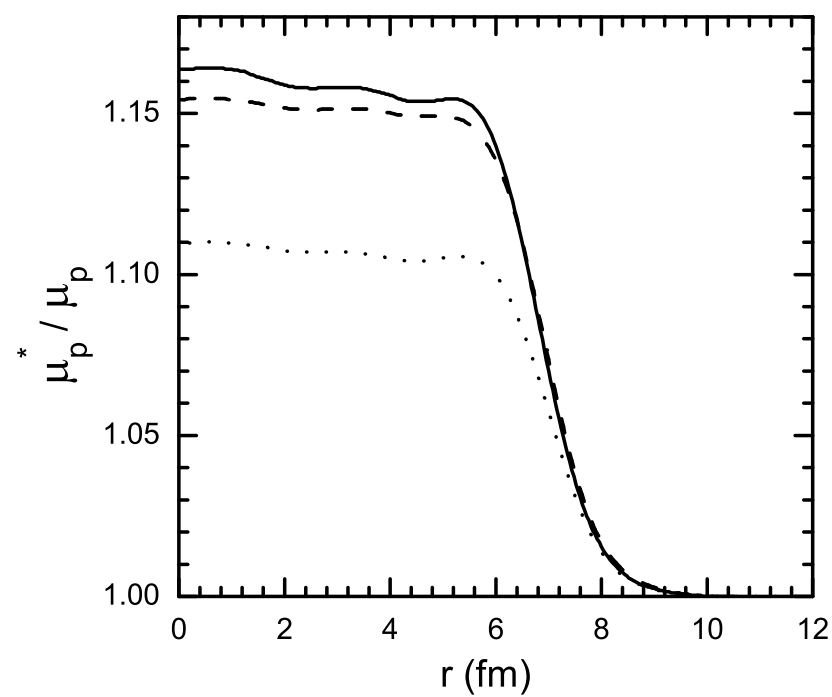

FIG. 10: The ratio of the proton magnetic moment in ${ }^{208} \mathrm{~Pb}$ to that in free space as a function of radial coordinate $r$. The lines are labeled as in Fig. 9 , 